\title{
Effects of Reciprocal Treatment with Estrogen and Estrogen Plus Parathyroid Hormone on Bone Structure and Strength in Ovariectomized Rats
}

\author{
Victor Shen, ${ }^{\star \S}$ Richard Birchman, ${ }^{\star}$ Rong Xu, ${ }^{*}$ Mark Otter, ${ }^{\ddagger}$ DaDong Wu, ${ }^{\ddagger}$ Robert Lindsay, " and David W Dempster $\$$ \\ ${ }^{*}$ Regional Bone Center and ${ }^{\ddagger}$ Orthopedic Engineering Research Center, Helen Hayes Hospital, W. Haverstraw, New York 10993; and \\ ${ }^{\S}$ Departments of Pathology and "Medicine, College of Physicians and Surgeons, Columbia University, New York 10032
}

\begin{abstract}
Intermittent administration of PTH has been found to be an effective anabolic agent in cancellous bone. We have reported previously that combined treatment with PTH and estrogen in estrogen-deficient rats was beneficial in correcting established osteopenia. To determine if the beneficial effects of PTH therapy can be preserved by estrogen alone and whether PTH therapy can be effective in treating osteopenic subjects stabilized with estrogen, we have undertaken a "crossover" study in the rat model of estrogendeficiency induced osteopenia. Six-month-old female rats were ovariectomized and after 5 wk treated for 8 wk with vehicle, $30 \mu \mathrm{g} / \mathrm{kg}$ per day of $\mathrm{rPTH}(1-34)$ plus $15 \mu \mathrm{g} / \mathrm{kg}$ per day of $17 \beta$-estradiol or $17 \beta$-estradiol alone. One group from each treatment regimen was then sacrificed and for an additional 8 weeks the remaining rats were $(a)$ maintained on their previous treatment; $(b)$ "crossed over" to their reciprocal treatment; or (c) administered vehicle only. At the end of this second 8-wk treatment period all rats were sacrificed. Bone mineral density of the distal femur, histomorphometric measurements of the proximal tibia and mechanical testing of the distal femur and selected vertebral bodies were performed. Our results demonstrated that $(a)$ the gains in bone mass, trabecular connectivity and mechanical strength induced by PTH can be maintained by estrogen alone, but are reversed when both agents are withdrawn; and $(b)$ rats with established osteopenia, maintained on estrogen treatment alone, can derive the full beneficial effects from the addition of PTH to the treatment at a later date. These data indicate that combined and/or sequential use of antiresorptive and anabolic agents may be a promising approach to the treatment of osteoporosis. (J. Clin. Invest. 1995. 96:2331-2338.) Key words: ovariectomy • osteoporosis • PTH • estrogen • mechanical competency
\end{abstract}

\section{Introduction}

Antiresorptive agents have long been used successfully to prevent bone loss associated with postmenopausal osteoporosis. However, these agents have not been able to restore lost bone

Address correspondence to Victor Shen, Ph.D., Regional Bone Center, Helen Hayes Hospital, W. Haverstraw, New York 10993. Phone: 9149473000 ext. 3843; FAX: 914-947-2485.

Received for publication 9 March 1995 and accepted in revised form 18 July 1995.

J. Clin. Invest.

(C) The American Society for Clinical Investigation, Inc.

0021-9738/95/11/2331/08 \$2.00

Volume 96, November 1995, 2331-2338 mass or microarchitecture (1). The search for an anabolic agent that is capable of reversing this bone loss has received substantial attention in recent years. Several agents, such as fluoride $(2,3)$, intermittent PTH in humans (4-7) and in rats (8-20), $\mathrm{PGE}_{2}$ (21-24), nandrolone decanoate (25), TGF-b2 (26-27), and IGF-1 $(28-34)$ in rats, have shown promise to fulfill such a role. We $(19,35)$, and others $(15-17)$, have recently shown that a treatment regimen combining antiresorptive and anabolic agents can halt further cancellous bone loss and restore some of the lost bone mass and structure, whether given in a preventive or curative mode. Long-term parenteral administration of an anabolic agent can be inconvenient, as well as a source of discomfort for the patient and thus results in reduced compliance. In long term usage, there also exist the possible problems of toxicity, loss of effectiveness, and economic considerations. Studies in rats have demonstrated a rapid disappearance of newly acquired bone when the anabolic agent is withdrawn and, therefore, continuous administration was needed to preserve the gain $(36,37)$. Jee and colleagues have proposed the use of an antiresorptive agent to maintain newly gained bone mass, induced by $\mathrm{PGE}_{2}$, in rats $(23,24)$. Since PTH has been used successfully in both humans (4-7) and rats $(8,9,11,13,14$, 18 ) to restore lost bone mass, we decided to examine whether the newly gained bone can be preserved by estrogen therapy alone.

In animal studies, PTH has been used to treat estrogen deficient rats by itself or concomitent with antiresorptive agents. This is somewhat different from the patients who are treated at osteoporosis clinics as many of them are often on hormone replacement therapy already. In the present study, we undertook an investigation to determine if the benefical effects of PTH can be realized after prolonged use of estrogen to stablize the skeleton in an animal model of postmenopausal osteoporosis.

\section{Methods}

Animals. Virgin female Sprague-Dawley rats were purchased from Harlan Sprague-Dawley, Inc. (Indianapolis, IN) and maintained at the Animal Research Facility of the Helen Hayes Hospital for an additional two months before the start of the experiment. They were housed in a room maintained at $20^{\circ} \mathrm{C}$ on a $12 \mathrm{~h}$ light $/ 12 \mathrm{~h}$ dark cycle. Bilateral ovariectomy ( Ovx) or sham operations were performed at the outset of the experiment when the rats were 6 mo of age and weighed $\sim 285$ grams. The Ovx animals were fed 15 grams per day of Purina laboratory rodent chow (Ralston Purina Co., St. Louis, MO), the mean food intake of the sham-operated animals, to prevent the hyperphagia associated with Ovx in rats. The experimental protocol was approved by the Institutional Animal Care and Use Committee at Helen Hayes Hospital.

Experimental protocols. 6-mo-old rats were ovariectomized then, after $5 \mathrm{wk}$, treated daily with intermittent sc injections of $30 \mu \mathrm{g} / \mathrm{kg}$ per day of rat PTH (1-34) (Bachem, Torrance, CA) plus $15 \mu \mathrm{g} / \mathrm{kg}$ per day of $17 \beta$-estradiol (Sigma Chem. Co., St. Louis, MO), 17 $\beta$-estradiol alone or vehicle for $8 \mathrm{wk}$. One group from each treatment regimen was sacrificed and for an additional $8 \mathrm{wk}$ the remaining rats were: $(a)$ 


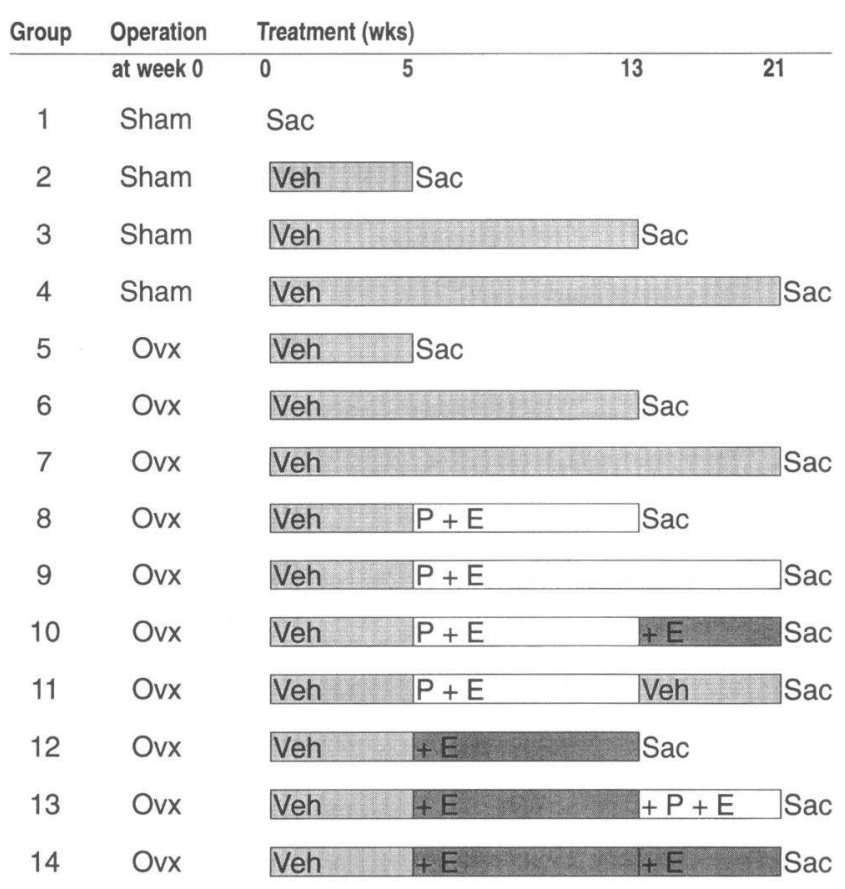

Veh = vehicle; $\mathbf{P}=\operatorname{rat}(1-34) \mathrm{PTH} @ 30 \mu \mathrm{g} / \mathrm{kg} / \mathrm{d}$;

$E=17 ß$-estradiol @ $15 \mu \mathrm{g} / \mathrm{kg} / \mathrm{d} ;$ Sac = sacrifice

Figure 1. Experimental Protocol.

continued on the same treatment; $(b)$ "crossed over" to their reciprocal treatment (i.e., PTH+estrogen to estrogen only or estrogen only to PTH+estrogen), or (c) given vehicle only. Each group consisted of 68 animals. The detailed protocol is as shown in Fig. 1. At the end of the second $8 \mathrm{wk}$ treatment period all rats were sacrificed. Urine was collected via metabolic cages over the $24 \mathrm{~h}$ before sacrifice. The rats were exsanguinated via the abdominal aorta under chloral hydrate anesthesia.

Bone mineral density. Bone mineral density of excised femurs was measured by dual energy $x$-ray absorptiometry (QDR-1000, Hologic, Waltham, MA) under $5 \mathrm{~cm}$ of $70 \%$ alcohol with a 0.025 inch collimator at high resolution mode. $25 \%$ of the femur, as measured from the distal end, which is rich in cancellous bone was analyzed. Triplicate determinations of five different femurs, with a new placement of the bone after each determination, showed a coefficient of variation at $2.5 \%$.

Osteocalcin and pyridinoline assays. The rat serum osteocalcin concentration was measured with a commercial radioimmunoassay kit (Biomedical Technologies Inc., Stoughton, MA) using rat osteocalcin as standard and a region specific domain of goat anti-rat osteocalcin antibody. Urinary pyridinoline and creatinine were measured by an ELISA kit from Metra Biosystems (Palo Alto, CA). Intra-assay coefficients of variation for rat osteocalcin and pyridinoline were 6 and 12\%, respectively.

Histomorphometry and trabecular strut analysis. Excised right tibias were dehydrated, embedded undecalcified in methyl methacrylate and sectioned longitudinally using a Jung Ultracut microtome (ReichertJung, Heidelberg, Germany). From the center of the tibias, 4-mm nonconsecutive longitudinal sections were obtained and stained by the Goldner's trichrome method. Cancellous bone volume ( $\mathrm{Cn}-\mathrm{BV} / \mathrm{TV})$, trabecular perimeter ( $\mathrm{Tb} . \mathrm{Pm})$ and trabecular thickness ( Tb.Th) were measured using a direct tracing method in an interactive measuring system (Optomax VIDS IV, Analytical Measurement Systems, Optomax Inc., Hollis, $\mathrm{NH}$ ). Using a 6.3 objective, the section was viewed on a television monitor at a total magnification of 140 . Two fields from each section, situated equidistant from the cortex and $1 \mathrm{~mm}$ distal to the lowest point of the growth plate, were used in the measurements. Each field was
$1.34 \mathrm{~mm}^{2}$ in area. Four sections were measured from each rat. Trabecular number ( Tb.N) and separation ( Tb.Sp) were calculated according to Parfitt et al. (39). For the strut measurements, definitions of the parameters were a modification (17) of those described previously by Garrahan et al. (40) and Mellish et al. (41). They included node number (N.Nd), terminus number (N.Tm), node to node strut length (Nd.Nd), node to terminus strut length (Nd.Tm) and terminus to terminus strut length (Tm.Tm), all expressed per $\mathrm{mm}^{2}$ of measured area. The measurements in the present study differed from previous ones in that each strut that extended beyond the border of the field was given a designation of Nd.Nd, Nd.Tm, or Tm.Tm, based on the identity of structures that were immediately outside the viewing field. Total strut length (TSL) is the sum of Nd.Nd, Nd.Tm and Tm.Tm.

Mechanical testing of the vertebral body and femur. Femurs from all seven groups and vertebrae from four groups collected at the last sacrifice were subjected to two types of mechanical testing, using a Materials Testing System (Model 810; MTS Systems Corp., Minneapolis, $\mathrm{MN}$ ) (a) Compression test of vertebral body. L5 vertebrae, with the two epiphyseal ends, posterior pedicle arch and spinous process removed, were compressed to failure at a displacement rate of $0.1 \mathrm{~mm}$ / $\mathrm{s}$ using a $2.5 \mathrm{kNewton}$ load cell (MTS Model 661.14A-03) as described by Mosekilde et al. (40). (b) Indentation test of the distal femur. An indenter was used to test the mechanical properties of the cancellous bone in the marrow cavity of the distal femur, using a modification of the methods used to determine mechanical strength of knee replacements in humans $(41,42)$. A 4-mm section of the distal femur was cut directly proximal to the femoral condyle with a low speed saw (Isomet, Buehler LTD, Lake Bluff, IL) under constant saline irrigation. A cylindrical indenter (with flat testing face) of $1.6 \mathrm{~mm}$ diameter, attached to a 11.4 $\mathrm{kg}$ load cell (MTS Model 3397-101) was applied to the center of the marrow cavity on the distal face of the section at a constant displacement velocity of $0.1 \mathrm{~mm} / \mathrm{s}$. The indenter was allowed to penetrate the cavity to a depth of $2 \mathrm{~mm}$ before load reversal.

Statistical analysis. The effects of treatment, treatment periods or aging were compared by the ANOVA method using Duncan's multiple group comparison procedure with SAS software (SAS Institute Inc, Cary, NC). A $P$ value of 0.05 was used to determine statistical significance.

\section{Results}

Beneficial effects of combined PTH and estrogen treatment; maintenance of acquired bone by estrogen treatment alone. As expected there was a significant decrease of $\mathrm{Cn}-\mathrm{BV} / \mathrm{TV}$ and Nd.Nd strut length in vehicle-treated Ovx rats (groups 5-7), when compared with Sham groups (groups 1-4) (Figs. 23 and see Table II). Combined PTH and estrogen treatment administered to Ovx rats (groups 8 and 9) after the rapid bone loss phase prevented further cancellous bone loss (Fig. 2) and also restored nearly all losses to the trabecular microarchitecture, as determined by Nd.Nd measurements (Fig. 3, Table I). The gain in cancellous bone volume was significant for the first 8 wk of treatment (group 8) but further treatment with the combination of PTH and estrogen (group 9) did not result in any additional increments in Cn-BV/TV or parameters of connectivity but did seem to increase the BMD of the distal femur (Figs. 2-3, Table I). Tb.Th increased in rats treated with PTH (Table I) but was unaffected by estrogen depletion (groups 57 ). With the combined PTH and estrogen treatment (groups 8-9), Tb.N and the parameters of connectivity (N.Nd, N.Nd/ N.Tm) were increased and disconnectivity parameters (Tb.Sp and $\mathrm{Tm} . \mathrm{Tm} / \mathrm{TSL}$ ) were decreased, when compared with vehicle treated Ovx rats (groups 5-7) (data not shown). This change was also seen in BMD measurements of the distal femur, a cancellous-rich region of the femur (Fig. 4, Table I). With- 


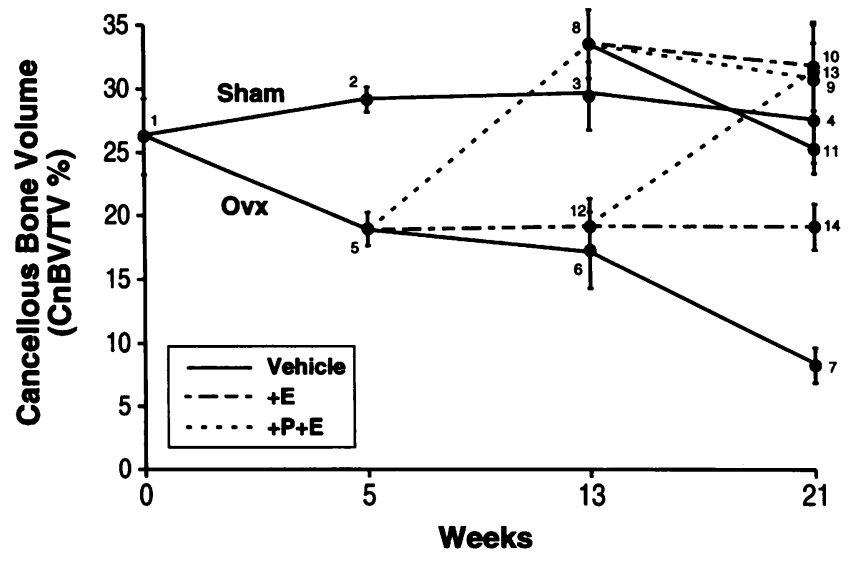

Figure 2. Effects of combined PTH plus estradiol and crossover treatments on cancellous bone volume at the proximal tibia. Numbered points represent group numbers. The sample variations are reported in $\mathrm{Ta}$ ble I.

drawal of both PTH and estrogen after 8 wk of treatment (group 11 ) resulted in significant decreases in bone mass and structure, whereas, maintaining treated animals on estrogen alone (group 10 ) protected the gains in bone mass and structure, when compared with a similarly treated group (group 8 ) before withdrawal.

PTH treatment can be used as an anabolic agent after bone loss has been previously stabilized by estrogen. In rats with established estrogen deficiency-induced bone loss, as expected, estrogen treatment was effective in preventing further bone loss (groups 12, 14 vs. group 5). These rats retained their Cn-BV/ $\mathrm{TV}$, connectivity parameters ( $\mathrm{Nd} . \mathrm{Nd}$ ) and bone mass in cancellous-rich regions of bone, when compared to their bone status at the initiation of treatment (group 5, Fig. 2-3, Table I). When PTH and estrogen were co-administered to Ovx rats with established osteopenia, after stabilization with estrogen for 8 wk (group 13), the combined treatment restored bone mass and structural parameters to the level of age-matched, Shamoperated animals (group 4) (Fig. 2-3, Table I).

Biochemical markers of bone turnover; osteocalcin and pyridinoline. Biochemical markers of turnover, osteocalcin and pyridinoline, showed an age-related decrease in Sham-operated animals (groups 1-4). Concentrations of each marker in Ovx rats treated with vehicle only were significantly increased when compared with age-matched Sham controls (group 5 vs. 2, group 6 vs. 3 and group 7 vs. 4, Table II). The groups of Ovx rats that received estrogen replacement (groups 8-10 and 1214), with or without concomitant or reciprocal PTH treatment, showed significantly lower levels of the two markers, when compared with vehicle treated, age-matched Ovx animals (groups 5-7) (Table II). Osteocalcin and pyridinoline levels returned to the elevated levels seen in untreated Ovx rats (group 7) in rats in which both PTH and estrogen treatment were withdrawn (group 11). The addition of PTH to the estrogen treatment resulted in a moderately higher level of osteocalcin than estrogen treatment alone, but the difference was not significant.

Mechanical strength is related to cancellous bone volume and connectivity parameters. In all groups sacrificed at week 21 , mechanical strength of the cancellous bone-rich distal femur, cortical bone-rich femoral diaphysis and vertebral bodies were

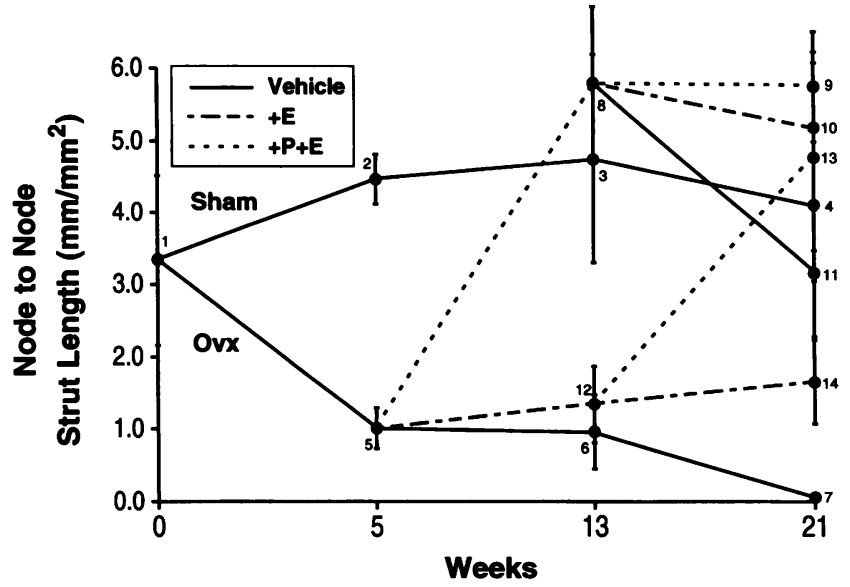

Figure 3. Effects of combined PTH plus estradiol and crossover treatments on node to node strut length at the proximal tibia. Numbered points represent group numbers. The sample variations are reported in Table I.

examined. This time period was employed in order to allow for significant bone loss in the vertebral bodies, as determined by Wronski et al. (45). Mechanical strength of the distal femur, using an indentation test that applied force directly into the marrow cavity, correlated with the BMD of the distal femur (initial max. load: $r=0.60, P<0.01$; stiffness: $r=0.32, P$ $<0.05$ ) and the proximal tibial Cn-BV/TV (initial max. load: $r=0.63, P<0.01$ ) but not with the diaphyseal femur BMD (initial max. load: $r=0.25$, NS; stiffness: $r=0.26$, NS). The compressive strength of the vertebral bodies showed similar results in that it correlated with the BMD of the distal femur (peak load: $r=0.59, P<0.01$; stiffness: $r=0.58, P<0.01$ ) but not with the BMD of the diaphyseal femur (peak load: $r$ $=0.37$, NS; stiffness: $r=0.49, P<0.05$ ). Thus, among cancellous-rich bones, the changes in the BMD and cancellous bone volume were proportionally reflected by the changes in the mechanical strength (Table III). The stiffness measurements from each group were similar to the load measurements.

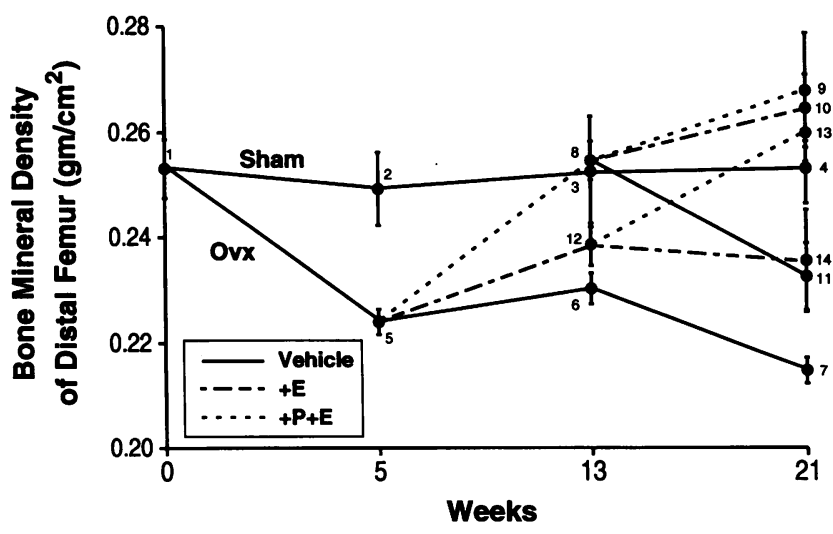

Figure 4. Effects of combined PTH plus estradiol and crossover treatments on bone mineral density at the distal femur. Numbered points represent group numbers. The sample variations are reported in Table I. 
Table IA. Histomorphometry and BMD Measurements

\begin{tabular}{|c|c|c|c|c|c|c|c|c|}
\hline \multirow[b]{2}{*}{ Group } & \multirow[b]{2}{*}{ Operation } & \multicolumn{3}{|c|}{ Treatment } & \multicolumn{3}{|c|}{ Histomorphometric Parameters } & \multirow{2}{*}{$\frac{\text { BMD }}{\text { Distal femur }}$} \\
\hline & & $0-5 \mathrm{wk}$ & $5-13 \mathrm{wk}$ & $13-21 \mathrm{wk}$ & Cn-BV/TV & Tb.Th & Nd.Nd & \\
\hline & & & & & $\%$ & $\mu m$ & $\mathrm{~mm} / \mathrm{mm}^{2}$ & $\mathrm{gm} / \mathrm{cm}^{2}$ \\
\hline 1 & Sham & & & & $26.2 \pm 3.0$ & $53.7 \pm 4.0$ & $3.34 \pm 1.18$ & $.2531 \pm .0056$ \\
\hline 2 & Sham & Veh & & & $29.1 \pm 1.0$ & $54.2 \pm 1.3$ & $4.47 \pm 0.35$ & $.2492 \pm .0070$ \\
\hline 3 & Sham & Veh & Veh & & $29.4 \pm 2.7$ & $58.9 \pm 3.4$ & $4.75 \pm 1.45$ & $.2525 \pm .0106$ \\
\hline 4 & Sham & Veh & Veh & Veh & $27.5 \pm 3.3$ & $69.4 \pm 5.4$ & $4.11 \pm 1.06$ & $.2532 \pm .0067$ \\
\hline 5 & Ovx & Veh & & & $18.9 \pm 1.3$ & $57.1 \pm 5.2$ & $1.01 \pm 0.28$ & $.2240 \pm .0024$ \\
\hline 6 & Ovx & Veh & Veh & & $17.3 \pm 3.0$ & $59.0 \pm 5.0$ & $0.96 \pm 0.51$ & $.2303 \pm .0029$ \\
\hline 7 & Ovx & Veh & Veh & Veh & $8.2 \pm 1.4$ & $58.2 \pm 4.3$ & $0.06 \pm 0.03$ & $.2148 \pm .0025$ \\
\hline 8 & Ovx & Veh & $P+E$ & & $33.5 \pm 2.7$ & $70.8 \pm 2.8$ & $5.80 \pm 1.06$ & $.2546 \pm .0037$ \\
\hline 9 & Ovx & Veh & $P+E$ & $P+E$ & $30.7 \pm 2.9$ & $74.9 \pm 5.3$ & $5.76 \pm 0.76$ & $.2680 \pm .0108$ \\
\hline 10 & Ovx & Veh & $P+E$ & E & $31.8 \pm 3.5$ & $72.2 \pm 2.0$ & $5.20 \pm 1.04$ & $.2647 \pm .0063$ \\
\hline 11 & Ovx & Veh & $P+E$ & Veh & $25.3 \pm 1.9$ & $68.0 \pm 2.3$ & $3.16 \pm 0.88$ & $.2324 \pm .0063$ \\
\hline 12 & Ovx & Veh & $\mathrm{E}$ & & $19.1 \pm 2.2$ & $62.8 \pm 3.1$ & $1.34 \pm 0.53$ & $.2386 \pm .0040$ \\
\hline 13 & Ovx & Veh & $\mathrm{E}$ & $P+E$ & $31.4 \pm 3.7$ & $75.2 \pm 3.5$ & $4.78 \pm 1.31$ & $.2600 \pm .0055$ \\
\hline 14 & Ovx & Veh & $\mathrm{E}$ & E & $19.1 \pm 1.8$ & $58.9 \pm 2.3$ & $1.65 \pm 0.58$ & $.2356 \pm .0097$ \\
\hline
\end{tabular}

\section{Discussion}

The published literature describing the use of anabolic agents to restore previously lost bone contains some very promising results $(13,16,17,19,24)$ but withdrawal of anabolic agent treatment has been shown to result in the rapid loss of all bone gained. To anticipate the need to terminate anabolic treatment when the bone mass has returned to a point where fracture risk is substantially reduced or where treatment has to be discontinued for any reason, we have studied the efficacy of using estrogen alone to maintain the increased bone mass. In the present study, we have employed an animal model of postmenopausal osteoporosis in a "crossover" study to determine whether estrogen treatment alone can maintain improvements in bone mass and microarchitecture and whether this anabolic treatment can be applied to bone which was previously stabilized by estrogen.

We, and others, have previously demonstrated that combined estrogen and PTH treatment can be effective both in preventing and treating estrogen deficiency-induced bone loss $(15-17,19)$ due to its ability to increase connectivity parameters (19) and thicken trabecular plates. However, it was shown that the newly gained bone mass would be lost if the treatment was not continued $(35,36,46)$. The idea of using an antiresorptive agent to preserve the bone gained from the use of anabolic agents has only recently been proposed. Jee and his colleagues demonstrated that one can preserve $\mathrm{PGE}_{2}$-induced bone gain by administering risedronate $(23,24)$. Physical exercise (46) and pamidronate (47) were shown to maintain PTHstimulated increases in bone mass in ovariectomized rats after

Table IB. Multiple Statistical Comparisons of Histomorphometric and Bone Mineral Density Parameters

\begin{tabular}{|c|c|c|c|c|c|c|c|c|c|c|c|c|c|c|}
\hline Group no. & 1 & 2 & 3 & 4 & 5 & 6 & 7 & 8 & 9 & 10 & 11 & 12 & 13 & 14 \\
\hline 1 & & & & b & ad & ad & acd & $a b c$ & b & b & b & & b & \\
\hline 2 & & & & b & acd & acd & acd & b & b & b & b & $\mathrm{ac}$ & b & ac \\
\hline 3 & & & & & acd & acd & acd & $\mathrm{b}$ & b & b & & $\mathrm{ac}$ & b & $\mathrm{ac}$ \\
\hline 4 & & & & & abcd & acd & acd & & & & & $\mathrm{ac}$ & & $\mathrm{a}$ \\
\hline 5 & & & & & & & $\mathrm{ac}$ & abcd & abcd & abcd & & & abcd & \\
\hline 6 & & & & & & & $\mathbf{a}$ & abcd & abcd & abcd & & & abcd & \\
\hline 7 & & & & & & & & abcd & abcd & abcd & $\mathrm{ac}$ & ad & abcd & ad \\
\hline 8 & & & & & & & & & & & $\mathrm{a}$ & $\mathrm{ac}$ & & $a b c$ \\
\hline 9 & & & & & & & & & & & d & abcd & & $a b c$ \\
\hline 10 & & & & & & & & & & & d & acd & & abcd \\
\hline 11 & & & & & & & & & & & & & d & b \\
\hline 12 & & & & & & & & & & & & & abcd & \\
\hline 13 & & & & & & & & & & & & & & abcd \\
\hline 14 & & & & & & & & & & & & & & \\
\hline
\end{tabular}

It is shown at statistical significant with $P$ value set at 0.05 in the Duncan's multiple range test of the ANOVA. $a, P<0.05$ for Cn-BV/TV; $b, P$ $<0.05$ for Tb.Th; $c, P<0.05$ for Nd.Nd; $d, P<0.05$ for BMD at distal femur. 


\begin{tabular}{|c|c|c|c|c|c|c|}
\hline \multirow[b]{2}{*}{ Group } & \multirow[b]{2}{*}{ Operation } & \multicolumn{3}{|c|}{ Treatment } & \multicolumn{2}{|c|}{ Marker concentration } \\
\hline & & $0-5 \mathrm{wk}$ & $5-13 \mathrm{wk}$ & $13-21 \mathrm{wk}$ & Osteocalcin & Pyridinoline \\
\hline & & & & & $n g / m l$ & $\mathrm{nmol} / \mathrm{mmol}$ \\
\hline 1 & Sham & & & & $25.1 \pm 1.4$ & $356 \pm 30$ \\
\hline 2 & Sham & Veh & & & $22.2 \pm 1.4$ & ND \\
\hline 3 & Sham & Veh & Veh & & $17.4 \pm 1.5$ & ND \\
\hline 4 & Sham & Veh & Veh & Veh & $14.0 \pm 0.9$ & $226 \pm 30$ \\
\hline 5 & Ovx & Veh & & & $30.3 \pm 1.2$ & $943 \pm 79$ \\
\hline 6 & Ovx & Veh & Veh & & $24.8 \pm 2.4$ & $428 \pm 46$ \\
\hline 7 & Ovx & Veh & Veh & Veh & $22.9 \pm 1.7$ & $300 \pm 42$ \\
\hline 8 & Ovx & Veh & $P+E$ & & $13.6 \pm 1.0$ & $180 \pm 8$ \\
\hline 9 & Ovx & Veh & $P+E$ & $P+E$ & $11.1 \pm 1.5$ & $131 \pm 11$ \\
\hline 10 & Ovx & Veh & $P+E$ & $\mathrm{E}$ & $10.6 \pm 1.5$ & $219 \pm 29$ \\
\hline 11 & Ovx & Veh & $P+E$ & Veh & $24.5 \pm 1.6$ & $451 \pm 31$ \\
\hline 12 & Ovx & Veh & $\mathrm{E}$ & & $9.0 \pm 0.8$ & $164 \pm 13$ \\
\hline 13 & Ovx & Veh & E & $P+E$ & $11.4 \pm 2.3$ & $176 \pm 24$ \\
\hline 14 & Ovx & Veh & $\mathrm{E}$ & $\mathrm{E}$ & $8.9 \pm 1.1$ & $117 \pm 15$ \\
\hline
\end{tabular}

PTH withdrawal. Our present results provide firm support, in an animal model, for the efficacy of using an anabolic agent, such as PTH, to induce improvement in bone mass, microstructural and mechanical integrity and that such improvements can be maintained with estrogen.

Although anabolic agents such as PTH have been shown to be effective in treating established osteopenia in rats $(13,19)$, there has not been a previous study where anabolic treatment is initiated after established osteoporosis had been stabilized with estrogen. We have demonstrated here that the anabolic effects of PTH remain even after extensive prior use of estrogen. This result is in agreement with our ongoing human study, where PTH is being given to osteoporotic women who have been and continue to receive estrogen (38).

The question of whether the anabolic effects of PTH plateau has not been thoroughly answered. It is important because it will determine the extent of the beneficial effects one can expect from such treatment. Our findings indicate that there was little change in cancellous bone volume in the second $8 \mathrm{wk}$ of PTH plus estrogen treatment and this would support the idea that there, indeed, is a plateau in the action of PTH on cancellous bone. Three excellent articles describing temporal aspects of the anabolic action of PTH provided us with useful information (15-17). Mosekilde et al. using a longitudinal scanning method showed that changes in total body mineral content, including both cortical and cancellous bones, plateaued 12 to 15 wk after PTH treatment began. However, it took only 5-10 wk to reach the same plateau when PTH plus estrogen treatment was administered (15). The implication of these findings is that combined PTH and estrogen treatment could result in more rapid anabolic

Table IIB. Multiple Statistical Comparisons of Biochemical Parameters

\begin{tabular}{|c|c|c|c|c|c|c|c|c|c|c|c|c|c|c|}
\hline Group no. & 1 & 2 & 3 & 4 & 5 & 6 & 7 & 8 & 9 & 10 & 11 & 12 & 13 & 14 \\
\hline 1 & & & a & a & b & & & $a b$ & $a b$ & a & & $a b$ & $a b$ & $a b$ \\
\hline 2 & & & & a & a & & & a & $\mathrm{a}$ & a & & a & $\mathrm{a}$ & a \\
\hline 3 & & & & & a & a & & & a & a & & a & a & a \\
\hline 4 & & & & & $a b$ & $a b$ & a & & & & $a b$ & & & \\
\hline 5 & & & & & & b & b & $a b$ & $a b$ & $a b$ & $a b$ & $a b$ & $a b$ & $a b$ \\
\hline 6 & & & & & & & b & $a b$ & $a b$ & $a b$ & & $a b$ & $a b$ & $a b$ \\
\hline 7 & & & & & & & & $\mathrm{a}$ & $a b$ & $\mathrm{a}$ & b & $\mathbf{a}$ & $\mathbf{a}$ & $a b$ \\
\hline 8 & & & & & & & & & & & $a b$ & & & \\
\hline 9 & & & & & & & & & & & $\mathrm{ab}$ & & & \\
\hline 10 & & & & & & & & & & & $a b$ & & & \\
\hline 11 & & & & & & & & & & & & $a b$ & $a b$ & $a b$ \\
\hline 12 & & & & & & & & & & & & & & \\
\hline 13 & & & & & & & & & & & & & & \\
\hline 14 & & & & & & & & & & & & & & \\
\hline
\end{tabular}

It is shown as statistical significant with $P$ value set at 0.05 in the Duncan's multiple range test of the ANOVA. $a, P<0.05$ for Osteocalcin, $b, P$ $<0.05$ for Pyridinoline. 


\begin{tabular}{|c|c|c|c|c|c|c|c|c|}
\hline \multirow[b]{3}{*}{ Group } & \multirow[b]{3}{*}{ Operation } & \multicolumn{3}{|c|}{ Treatment } & \multicolumn{4}{|c|}{ Mechanical Testing } \\
\hline & & \multirow[b]{2}{*}{$0-5 \mathrm{wk}$} & \multirow[b]{2}{*}{$5-13 \mathrm{wk}$} & \multirow[b]{2}{*}{$13-21 \mathrm{wk}$} & \multicolumn{2}{|c|}{ Indentation of distal femur } & \multicolumn{2}{|c|}{ Compression of vertebral body } \\
\hline & & & & & Init. max load & Stiffness & Load & Stiffness \\
\hline & & & & & newton & newton $/ \mathrm{mm}$ & newton & newton $/ \mathrm{mm}$ \\
\hline 4 & Sham & Veh & Veh & Veh & $36.6 \pm 6.1$ & $632 \pm 95$ & $348 \pm 32$ & $3588 \pm 274$ \\
\hline 7 & Ovx & Veh & Veh & Veh & $6.0 \pm 1.4$ & $172 \pm 40$ & $263 \pm 17$ & $2805 \pm 160$ \\
\hline 9 & Ovx & Veh & $P+E$ & $P+E$ & $50.6 \pm 15.2$ & $660 \pm 100$ & $393 \pm 26$ & $4242 \pm 309$ \\
\hline 10 & Ovx & Veh & $P+E$ & $\mathrm{E}$ & $50.2 \pm 12.9$ & $604 \pm 55$ & ND & ND \\
\hline 11 & Ovx & Veh & $P+E$ & Veh & $20.2 \pm 3.9$ & $326 \pm 59$ & ND & ND \\
\hline 13 & Ovx & Veh & E & $P+E$ & $43.2 \pm 10.9$ & $625 \pm 65$ & $375 \pm 33$ & $4128 \pm 334$ \\
\hline 14 & Ovx & Veh & $\mathrm{E}$ & $\mathrm{E}$ & $23.1 \pm 9.9$ & $376 \pm 122$ & ND & ND \\
\hline
\end{tabular}

action in cancellous bone than with PTH alone, and therefore, the maximum benefit was achieved earlier. One study, by Wronski et al, produced similar results as in our study. They demonstrated that cancellous bone in the proximal tibia showed a rapid PTH plus estrogen-induced increase of cancellous bone volume in the first $5 \mathrm{wk}$, after which the rate of increase abated, though it did not plateau. PTH treated rats, however, showed a definite plateau $10 \mathrm{wk}$ after commencement of treatment (16). Though the results from the various studies were not exactly the same they are generally in line with the idea that there is little change in cancellous bone volume beyond $8 \mathrm{wk}$ of PTH plus estrogen treatment, as we have reported. One should note three things (a) this plateau does not indicate that additional treatment periods would not be beneficial. Withdrawal of PTH treatment, without anti-resorptive agent intervention, has been shown to result in a rapid loss of the gained cancellous bone $(36) ;(b)$ very few determinants of this plateau effect, such as initial bone mass, effects at different skeletal sites, doses or frequency of treatment have yet to be determined; and $(c)$ little is known about the possibility of a second plateau resulting from renewed or cyclical treatment.

Wronski and Yen showed that cortical bone area, though not total bone area, at the tibial diaphysis continued to increase

Table IIIB. Multiple Statistical Comparisons of Mechanical Strength Testing Parameters

\begin{tabular}{|c|c|c|c|c|c|c|c|}
\hline Group no. & 4 & 7 & 9 & 10 & 11 & 13 & 14 \\
\hline 4 & & $b, c$ & & & b & . & b \\
\hline 7 & & & $\mathrm{a}, \mathrm{b}, \mathrm{c}, \mathrm{d}$ & $a, b$ & & $a, b, c, d$ & \\
\hline 9 & & & & & b & & b \\
\hline 10 & & & & & b & & \\
\hline 11 & & & & & & b & \\
\hline 13 & & & & & & & b \\
\hline 14 & & & & & & & \\
\hline
\end{tabular}

It is shown as statistical significant with $P$ value set at 0.05 in the Duncan's multiple range test of the ANOVA. $a, P<0.05$ for Init. max. load in the indentation test in the marrow cavity of distal femur; $b, P$ $<0.05$ for Stiffness in the indentation test in the marrow cavity of distal femur; $c, P<0.05$ for Load in compression test of vertebral body; $d, P<0.05$ for Stiffness in compression test of vertebral body. throughout $15 \mathrm{wk}$ of treatment similarly regardless if the rats were treated with PTH alone or with PTH plus estrogen. (17). This may be the reason why we observed a small increase in the BMD at the distal femur between 8 and 16 wk of PTH plus estrogen treatment. A small increase of BMD was also found when estrogen was given during weeks 5-13 in Ovx rats or weeks $13-21$ in rats previously treated with PTH plus estrogen. This could result from a transient reduction in the number of resorption cavities in Ovx rats and, possibly the continued anabolic action of PTH on cortical bone in PTH plus estrogen treated rats. One should also be aware that the complicated effects of estrogen-induced changes in cortical dimensions, such as expansion of the marrow cavity and periosteal surface, may affect the BMD measurements as well. Further studies will be needed to address the changes which occurred in cortical bone.

Bone mass has often been used as a surrogate to evaluate bone fragility. However, a direct method of testing the mechanical strength of the bone could provide more relevant information about its structural integrity (48). Bone strength is influenced by many factors, amongst them the content of organic and mineral phases and the internal architecture of the bone. The architecture of cancellous bone may be a particularly important contributor to its mechanical strength and this feature cannot be adequately addressed by bone mass measurements alone. We performed a series of mechanical testing techniques that were designed to be particularly indicative of the structural integrity in both axial (vertebral body) and appendicular (distal femur) bones. The high correlation between BMD, Cn-BV/TV and mechanical strength indicates that these determinants of bone mass can be a suitable surrogate for mechanical strength in this model. The high correlation between connectivity and disconnectivity parameters and mechanical strength also suggests that changes in the integrity of the trabecular lattice strongly influence the ability to support mechanical load. Though better studied in humans $(49,50)$, the validity of the relationship between cancellous-rich rat vertebral bone compressive strength and density in rats has been observed in group $(15,51)$ and individual (52) comparisons. Using an indentation test similar to the one employed in the present study, Martin (53) showed comparable changes in ash mass and penetration strength between control and experimental groups of rats at the distal femur and the proximal tibia. Thus, both methods of measuring cancellous bone strength are appropriate for future experimentation in rats. Biochemical markers, such as osteocalcin and pyridinoline, 
have been used extensively in the study of bone turnover. Using a rat specific osteocalcin radioimmunoassay and ELISA measurements of urinary pyridinoline, we showed that there was an age-related decrease in the level of these two markers. Estrogen deficiency, as a result of ovariectomy, significantly increased the level of both markers but the age-related decline was still evident in the estrogen deficient animals. Estrogen administration reversed the increase in turnover markers seen in ovariectomized animals and the markers rose again when estrogen was withdrawn. These results are very similar to the findings in human studies after oophorectomy (54) where biochemical bone turnover markers were elevated immediately after surgery and the elevation of these markers was abated after the initiation of estrogen replacement therapy. Osteocalcin, a bone formation marker, was found to be higher, although not significantly, in each of our PTH plus estrogen treated groups. The lack of statistical significance in these comparisons could result from ( $a$ ) the lack of sufficient sample size, $(b)$ insufficient sensitivity of the assay or, $(c)$ the percentage of osteocalcin released from osteoblasts into the circulation may be altered as a result of PTH administration so that more osteocalcin molecules were deposited into the newly formed bone. A different method of study will be needed to address these issues. In addition, one cannot rule out the possible influences on the markers of longitudinal or radial bone growth, as the skeletons of these animals were still growing, albeit, very slowly.

There are still questions which need to be answered before the anabolic action of PTH can be applied as a clinical tool. The use of animal models can provide a screening process to determine the effectiveness of these approaches. In this study, we have provided experimental data in support of the feasibility of using estrogen, an anti-resorptive agent, to preserve the bone gained by PTH treatment as well as the successful initiation of anabolic treatment after bone loss has been stabilized by estrogen. With further refinement, this type of sequential treatment with anti-resorptive and anabolic agents could be a promising approach for the future treatment of postmenopausal osteoporosis.

\section{Acknowledgments}

This study was supported by National Institutes of Health grant number AR-39191.

\section{References}

1. Christiansen, C. 1992. Prevention and treatment of osteoporosis: a review of current modalities. Bone. 13:S35-S39.

2. Kleerekoper, M., E. L. Peterson, D. A. Nelson, E. Philips, M. A. Schork, B. C. Tilley, and A. M. Parfitt. 1991. A randomized trial of sodium fluoride as a treatment for postmenopausal osteoporosis. Osteoporosis Int. 1:155-161.

3. Riggs, B. L., E. Seemon, S. F. Hodgeson, D. R. Taves, and W. M. O'Fallon. 1982. Effect of the fluoride/calcium regimen on vertebral fracture occurrence in postmenopausal osteoporosis. N. Engl. J. Med. 306:446-450.

4. Bradbeer, J. N., M. Arlot, J. Reeve, and P. J. Meunier. 1992. Treatment of osteoporosis with parathyroid peptide (hPTH 1-34) and estrogen: increase in volumetric density of iliac cancellous bone may depend on reduced trabecular spacing as well as increased thickness of packets of newly formed bone. Clin. Endocrinol. 37:282-289.

5. Hesch, R. D., U. Busch, M. Prokop, G. Delling, and E. F. Rittinghaus 1989. Increase of vertebral density by combination therapy with pulsatile 1-38 hPTH and sequential addition of calcitonin nasal spray in osteoporotic patients. Calcif. Tissue Int. 44:176-180.

6. Hesp, R., P. Hulme, D. Williams, and J. Reeve. 1981. The relationship between changes in femoral bone density and calcium balance in patients with involutional osteoporosis treated with human PTH fragment 1-34. Metab. Bone Dis. Rel. Res. 2:331-334.

7. Reeve, J., P. J. Meunier, J. A. Parsons, M. Bernat, O. L. M. Bijvoet, P. Courpron, C. Edouard, L. Klenerman, R. M. Neer, J. C. Renier, D. Slovik, E. J. F. E. Vismans, and J. T. Potts. 1980. Anabolic effect of human PTH on trabecular bone in involutional osteoporosis: a multicenter trial. $\mathrm{Br}$. Med. J. 280:1340-1344.

8. Ejersted, C., T. T. Andreassen, H. Oxlund, P. H. Jorgensen, B. Bak, J. Haggblad, O. Torring, and M. Nilsson. 1993. Human parathyroid hormone (1-

$34)$ and (1-84) increase the mechanical strength and thickness of cortical bone in rats. J. Bone Miner. Res. 9:1097-1101.

9. Gunness-Hey, M., and J. M. Hock. 1984. Increased trabecular bone mass in rats treated with synthetic human PTH. Metab. Bone Dis. Rel. Res. 5:177181 .

10. Hock, J. M., I. Gera, J. Fonseca, and L. G. Raisz. 1988. Human PTH (1-34) increases bone in ovariectomized and orchidectomized rats. Endocrinol. 122:2899-2903.

11. Kalu, D. N., R. Echon, and B. W. Hollis. 1990. Modulation of ovariectomyrelated bone loss by parathyroid hormone in rats. Mech. Aging Dev. 56:49-62.

12. Liu, C. C., and D. N. Kalu. 1990. Human parathyroid hormone (1-34) prevents bone loss and augments bone formation in sexually mature ovariectomized rats. J. Bone Miner. Res. 5:973-981.

13. Liu, C. C., D. N. Kalu, E. Salerno, R. Echon, B. W. Hollis, and M. Ray. 1991. Preexisting bone loss associated with ovariectomy in rats is reversed by PTH. J. Bone Miner. Res. 6:1071-1080.

14. Mitlak, B. H., D. C. Williams, H. U. Bryant, D. C. Paul, and R. M. Neer. 1992. Intermittent administration of bovine PTH (1-34) increases serum 1,25dihydroxyvitamin D concentrations and spinal bone density in senile ( 23 month) rats. J. Bone Miner. Res. 7:479-484.

15. Mosekilde, L., C. C. Danielsen, and J. Gasser. 1994. The effect on vertebral bone mass and strength of long term treatment with antiresorptive agents (estrogen and calcitonin), human parathyroid hormone (1-38), and combination therapy, assessed in aged ovariectomized rats. Endocrinol. 134:2126-2134.

16. Wronski, T. J., C. F. Yen, and L. M. Dann. 1993. Parathyroid hormone is more effective than estrogen or bisphosphonate for restoration of lost bone mass in ovariectomized rats. Endocrinol. 132:823-831.

17. Wronski, T. J., and C. F. Yen. 1994. Anabolic effects of parathyroid hormone on cortical bone in ovariectomized rats. Bone. 15:51-58.

18. Oxlund, H., C. Ejersted, T. T. Andreassen, O. Torring, and M. H. L. Nilsson. 1993. Parathyroid hormone (1-34) and (1-84) stimulate cortical bone formation both from periosteum and endosteum. Calcif. Tissue Int. 53:394-399.

19. Shen, V., D. W. Dempster, R. Birchman, R. Xu, and R. Lindsay. 1993 Loss of cancellous bone mass and connectivity in ovariectomized rats can be restored by combined treatment with PTH and estradiol. J. Clin. Invest. 91:24792487.

20. Sogaard, C. H., T. J. Wronski, J. E. McOsker, and L. Mosekilde. 1994 The positive effect of parathyroid hormone on femoral neck bone strength in ovariectomized rats is more pronounced that of estrogen or bisphosphonates. Endocrinol. 134:650-657.

21. Akamine, T., W. S. Jee, H. Z. Ke, X. J. Li, and B. Y. Lin. 1992. PGE E2 prevents bone loss and adds extra bone to immobilized distal femoral metaphysis in female rats. Bone. 13:11-22.

22. Jee, W. S. S., S. Mori, S. Chan, and L. Chan. 1990. Prostaglandin E2 enhances cortical bone mass and activates intracortical bone remodeling in intact and ovariectomized female rats. Bone. 11:253-266.

23. Jee, W. S. S., L. Tang, H. Z. Ke, R. B. Setterberg, and D. B. Kimmel. 1993. Maintaining restored bone with bisphosphonate in the Ovx rat skeleton: dynamic histomorphometry of changes in bone mass. Bone. 14:493-498.

24. Tang, L. Y., W. S. S. Jee, H. Z. Ke, and D. B. Kimmel. 1992. Restoring and maintaining bone in osteopenic female rat skeleton: $\mathrm{I}$. Changes in bone mass and structure. J. Bone Miner. Res. 7:1093-1104.

25. Aerssens, J., R. V. Audeckercke, P. Geusens, L. P. Schot, A. A. H. Osman, and J. Dequeker. 1993. Mechanical properties, bone mineral content, and bone composition (collagen, osteocalcin, IGF-1) of rat femur: influence of ovariectomy and nandrolone decanoate (anabolic steroid) treatment. Calcif. Tissue. Int. 53:269-277.

26. Rosen, D., S. C. Miller, E. DeLeon, A. Y. Thompson, H. Bentz, M Mathews, and S. Adams. 1994. Systemic administration of recombinant TGF beta 2 stimulates parameters of cancellous bone formation in juvenile and adult rats. Bone. 15:355-359.

27. Kalu D. N., E. Salerno, Y. Higami, C. C. Liu, F. Ferraro, M. A. Salih and B. H. Arjmaridi. 1993. In vivo effects of TGF-b2 in ovariectomized rats. Bone Mineral. 22:209-220.

28. Ammann, P., R. Rizzoli, T. Shigematsu, and J. Bonjour. 1991. Chronic infusion of IGF-1 increases bone mineral density evaluated sequentially by dual energy $\mathrm{x}$-ray absorptiometry in ovariectomized osteopenic rats. J. Bone Miner. Res. 6 (Suppl 1) S218.

29. Ibbotson, K. J., C. M. Orcutt, S. M. D'Souza, C. L. Paddock, J. A. Arthur, M. L. Jankowsky, and R. W. Boyce. 1992. Contrasting effects of PTH and IGF- 
1 in an aged ovariectomized rat model of postmenopausal osteoporosis. J. Bone Miner. Res. 7:425-432.

30. Mueller, K , R Cortesi, D Modrowski, and P. J Marie. 1994. Stimulation of trabecular bone formation by insulin-like factor $\mathrm{I}$ in adult ovariectomized rats. Am. J. Physiol. 267:E1-E6.

31. Bagi, C. M., R. Brommage, L. DeLeon, S. Adams, D. Rosen, and A Sommer. 1994. Benefit of systemically administered rhIGF-1 and hIGF-1/IGFBP3 on cancellous bone in ovariectomized rats. J. Bone Miner. Res. 9:1301-1312.

32. Spencer. E. M., C. C. Liu, E. C. C. Si, and G. A. Howard. 1991. In vivo actions of IGF-1 on bone formation and resorption in rats. Bone. 12:21-26.

33. Ammann, P., R. Rizzoli, K. Mueller, D. Slosman, and J. P. Bonjour. 1994 IGF-1 and pamidronate increase bone mineral density in ovariectomized adult rats. Am. J. Physiol. 265:E770-E776.

34. Tobias, H., J. W. M. Chow, and T. J. Chambers. 1992. Opposite effects of IGF-1 on the formation of trabecular and cortical bone in adult female rats Endocrinol. 131:2387-2392.

35. Mellish, R. W. E., V. Shen, R. Birchman, X. W. Meng, W. Horbert, D. Dempster, and R. Lindsay. 1990. Quantitative analysis of trabecular bone in ovariectomized rats after intermittent administration of low dose human parathy roid hormone fragment (1-34) and 17b-estradiol. In Osteoporosis. C. Christiansen and K. Overgand, editors. Handestrykkeriet Aalborg APS, Aalborg, Denmark 1335-1337.

36. Gunness-Hey, M., and J. M. Hock. 1989. Loss of the anabolic effect of PTH on bone after discontinuation of hormone in rats. Bone. 10:447-452.

37. Ke, H. Z., X. J. Li, and W. S. S. Jee. 1991. Partial loss of anabolic effect of prostaglandin $\mathrm{E} 2$ on bone after its withdrawal in rats. Bone. 12:173-183.

38. Lindsay, R., F. Cosman, J. Nieves, D. W. Dempster, and V. Shen. 1993 A controlled clinical trial of the effects of $1-34 \mathrm{hPTH}$ in estrogen treated osteoporosis. J. Bone Miner. Res. 8 (Suppl 1): S130.

39. Parfitt, A. M., C. H. E. Matthews, A. R. Villanueva, M. Kleerekoper, B. Frame, and D. S. Rao. 1983. Relationships between surface, volume, and thickness of iliac trabecular bone in aging and in osteoporosis. Implications for the microanatomic and cellular mechanisms of bone loss. J. Clin. Invest. 72:1396-1409.

40. Garrahan, N. J., R. W. E. Mellish, and J. E. Compston. 1986. A new method for the two-dimensional analysis of bone structure in human iliac crest bone biopsies. J. Microsc. 142:341-349.

41. Mellish, R. W. E., M. W. Ferguson-Pell, G. V. B. Cochran, R. Lindsay, and D. W. Dempster. 1991. A new method for assessing two-dimensional cancellous bone structure: Comparison between iliac crest and lumbar vertebra. J. Bone Miner. Res. 6:689-696.

42. Mosekilde, L., C. C. Danielsen, and U. B. Knudsen. 1993. The effect of aging and ovariectomy on the vertebral bone mass and biomechanical properties of mature rats. Bone. 14:1-6.

43. Hvid, I., K. Andersen, and S. Olesen. 1984. The osteopenetrometer. Eng. Med. 13:73-78.

44. Hvid, I., and S. L. Hansen. 1985. Trabecular bone strength patterns at the proximal tibial epiphysis. J. Orthop. Res. 3:464-472.

45. Wronski, T. J., M. Cintron, and L. M. Dann. 1988. Temporal relationship between bone loss and increased bone turnover in ovariectomized rats. Calcif. Tissue Int. 43:179-183.

46. Yamamoto, N., H. E. Takahashi, T. Tanizawa, R. Fujimoto, T. Hara, and S. Tanaka. 1993. Maintenance of bone mass by physical exercise after discontinuation of intermittent hPTH (1-34) administration. Bone Miner. 23:333-342.

47. Cheng, P. T., C. Chan, and K. Muller. 1995. Cyclical treatment of osteoporotic ovariectomized adults rats with PTH (1-34) pamidronate. J. Bone Miner Res. 10:119-126.

48. Turner, C. H., and D. B. Burr. 1993. Basic biomechanical measurements of bone: a tutorial. Bone. 14:595-608.

49. Mosekilde, L. 1993. Vertebral structure and strength in vivo and in vitro (review). Calcif. Tissue Int. 53 (Suppl 1):S121-125.

50. Cody, D. D., S. A. Godkstein, M. J. Flynn, and E. B. Brown. 1991. Correlation between vertebral regional bone mineral density and whole bone fracture load. Spine. 16:146-154.

51. Mosekilde, L., C. H. Sogaard, J. E. McOsker, and T. J. Wronski. 1994 PTH has a more pronounced effect on vertebral bone mass and biomechanica competence than anit-resorptive agents (estrogen and bisphosphonate) assessed in sexually mature, ovariectomized rats. Bone. 15:401-408.

52. Katsumata, T., T. Nakamura, H. Ohnishi, and T. Sakurama. 1995. Intermittent cyclical etidronate treatment maintains the mass, structure and the mechanical property of bone in ovariectomized rats. J. Bone Miner. Res. 10:921-931.

53. Martin, R. B. 1990. Effects of simulated weightlessness on bone properties in rats. J. Biomechanics. 23:1021-1029.

54. Stepan, J. J., J. Pospichal, J. Presl, and V. Pacovsky. 1987. Bone loss and biochemical indices of bone remodeling in surgically induced postmenopausal women. Bone. 8:279-284. 